\title{
Artificial Neural Network Based Power Management for Smart Street Lighting Systems
}

\author{
Dr. S. Smys, \\ Professor, Department of Computer Science and Engineering, \\ RVS Technical Campus, \\ Coimbatore, India. \\ Email: smys375@gmail.com \\ Dr. Abul Basar, \\ Professor, Prince Mohammad Bin Fahd University, \\ Kingdom of Saudi Arabia. \\ Email: abashar@pmu.edu.sa \\ Dr. Haoxiang Wang, \\ Department of Electrical and Computer Engineering, \\ Cornell University, Ithaca, USA. \\ Email: wanghaoxiang1102@hotmail.com
}

\begin{abstract}
The modern ways to empower the ecofriendly people also insists the necessity to cutting back of energy consumption. The minimizing the energy consumption would in turn reduce the rate of carbon emission, resulting in a cleaner air quality and higher standard of living by paving way for a cleaner planet. The increasing demand on power requirement is also one of the important reason for minimizing the energy consumption. The paper tries to decrease the energy usage of the street light system as the lighting systems in the street does not have an efficient way of managing and controlling the power flow in them as they are incapable of taking into consideration the prevailing demands on the intensity of light. So the paper puts forwards the idea of power management in the smart street lighting to control efficiently the power consumption by comparing the intensity of the light with the weather conditions. The artificial neural networks is used in power management of the street lighting in the proposed method. The evaluation of the method show up with the results that produce the better management of the power and the reduced power usage in street lights.
\end{abstract}

Keywords: Energy Consumption, Smart Street Light, Artificial Neural Networks, Light Intensity, Weather Conditions

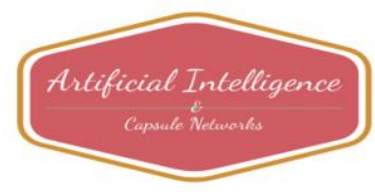


Journal of Artificial Intelligence and Capsule Networks (2020)

Vol.02/ No. 01

Pages: $42-52$

http://irojournals.com/aicn/

DOI: https://doi.org/10.36548/jaicn.2020.1.005

\section{Introduction}

The traditional lighting procedure followed in the streets utilizes very simple techniques that allow or stop the lights according to the intensity management of the light. The steps involved multitude of equipment's such as timers, nightfall sensors and astronomical clocks etc. these system were designed with the capability to switch on and off within a prescribed time. There are many other systems that were designed with (i) "light sensitive photocells" to switch on the light when the night falls and switch it off the lights on the dawn, (ii) the street lights designed with GPRS, to turn ON and as well OFF the lights according to the information's received based on the attributes of the location (Sun set, latitude, sun set and the longitude). (iii) An even advanced control system empowered with the LED lamps and enriched with the capability of diming the lights, schedule the on/off moments, and further regulate the light intensities.

Nowadays the LED with the improved performance are engaged in the street lights, to provide a more energy efficient control system to manage the power consumption, so the current work carried out in the paper scopes in developing a novel expert system engaged intelligent management of power in the street lightings. The artificial neural networks is used in the paper to support the power regulation and cut down the majority of energy usage and cost.

An efficient power management system for the regulating the power flow in the lighting in the street must be capable of minimizing the power consumption. So the proposed method utilizes the ANN in the process of regulating the power flow to minimize the usage of the energy.

The ANN which is also termed as a universal expert system is highly prominent in most of the application areas including engineering. It is mathematical frame work that is stimulated by the working and the basic arrangement of the biological neural network. They will learn on the basis of properly organized training patterns and generalize and use the information acquired in predictive calculations of original circumstances. Different types of NNs and different forms of their realization were recorded.

While software implementations are the most common, the field of ANN realized utilizing the Software as well as the hardware is swiftly growing. In some conditions, ANNs implemented in hardware can run faster and use less power compared to the software ones. We build new choices for area of transportable indication computing systems and apparatuses. The ANN typically entails crisscrossed collection of artificial-neurons, which are the fundamental essentials of these structures. The neuron is a multi-input and one output part. The outcomes of individual neurons are related to next neuron input, which form the general N-N. The

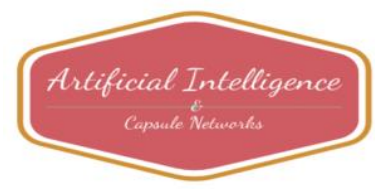


Journal of Artificial Intelligence and Capsule Networks (2020)

Vol.02/ No. 01

Pages: 42-52

http://irojournals.com/aicn/

DOI: https://doi.org/10.36548/jaicn.2020.1.005

arrangement of "ANN" is dictated linkage of the neurons and can divide the neurons into planes. The signals in input are transmitted to the neurons establishing links using the recognized weights.

The feed-forward is one of the predominant, neural networks. This neural network does not have uniformly dispersed links across the input and the output, and the flow of information's take place in one direction. The information's travel from input forwarded through the layers that are hidden and reaches output layers. The outcome of each layers is fed as input to its succeeding layers, the data got at the output plane is the complete neural network reaction. "The number of layers depends on the complexity of the problem being analyzed and must be experimentally calculated". The method of power management proposed utilizes the feed-forward type networks with the "Back Propagation Algorithm".

"Within the NN, the learning process is based on the concept that the NN's output values are compared to the correct response provided by the trainer to estimate the miscalculation. The error is fed back to the network, and the algorithm changes the weights of each link to minimize the error". Usually, next to reiterating this procedure for an appropriately hefty number of cycles in training, the system come together to some error. The figure. 1 below shows the basic structure of feed-forward network.

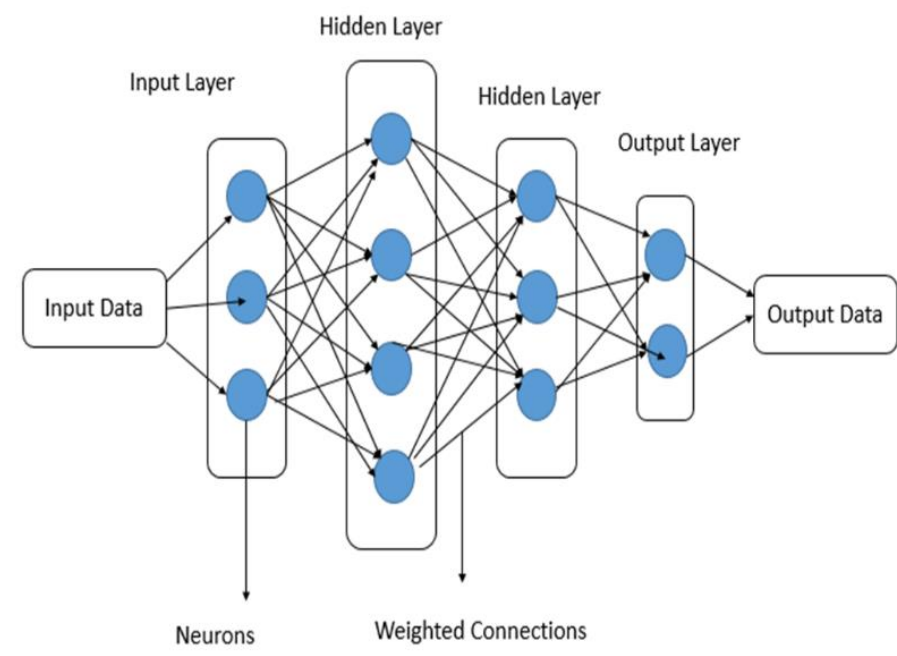

Figure.1 Basic Structure of Feed-Forward Network

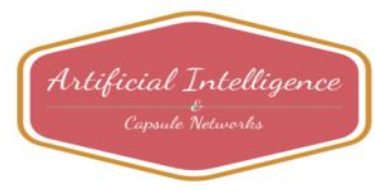


Journal of Artificial Intelligence and Capsule Networks (2020)

Vol.02/ No. 01

Pages: 42-52

http://irojournals.com/aicn/

DOI: https://doi.org/10.36548/jaicn.2020.1.005

The ANN utilized in the proposed method uses the procedure of "incrementally updating the networks weights." Along with alterations in the arrangement of patterns that were used in the training. The neuron weights are scaled up once the new vector is received for training. The error in the prediction $\left(P_{\text {err }}\right)$ is estimated using the following equation 1

$$
P_{e r r}=\sqrt{\frac{1}{x T P} \sum_{m=1}^{x L P} \sum_{k=1}^{i}\left(A_{m, k}-B_{m, K}\right)^{2}}
$$

While, $x T P$ is the average "number of training patterns", ' $\mathrm{i}$ ' layers in output. The training vectors are demarcated as ' $c$ ', the output vectors are denoted as ' $\mathrm{A}$ ' and the predictable solutions are denoted as ' $\mathrm{B}$ ' and ' $\mathrm{w}$ ' obviously the weight vector of the neuron given.

The proposed method is prepared with "related works in 2, the proposed work in 3 . Results evaluation in 4 and conclusion in 5".

\section{Related Works}

Canca, D., J et al [1] make effort to devise a "Traffic intensity forecast in urban networks using a multilayer perceptron." Ishak et al [2] performs the "Optimization of dynamic neural network performance for shortterm traffic prediction." Volosencu et al [3] proffers the "Hierarchical approach for intelligent lighting control in future urban environments."

Rea et al [4] describes the "Several views of metal halide and high-pressure sodium lighting for outdoor applications." Pamuła, Teresa et al [5] has put forth the "Road traffic parameters prediction in urban traffic management systems using neural networks."

Saravanan, K et al [6] proposed an "Fuzzy controller design of lighting control system by using VI package." Ramadhani, et al [7] devised an "Optimization of standalone street light system with consideration of lighting control."

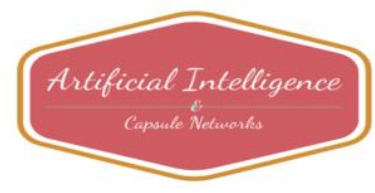


Journal of Artificial Intelligence and Capsule Networks (2020)

Vol.02/ No. 01

Pages: 42-52

http://irojournals.com/aicn/

DOI: https://doi.org/10.36548/jaicn.2020.1.005

Kumar, $\mathrm{R}$ et al [8] in his paper elaborates "A novel report on architecture, protocols and applications in Internet of Things (IoT)." Raj, Jennifer S. et al [9] conducts "A Comprehensive Survey on the Computational Intelligence Techniques and Its Applications."

Bestak, et al [10] performed a "Big Data Analytics for Smart Cloud-Fog Based Applications." Koresh, M. H., et al [11] has proposed a "Computer vision based traffic sign sensing for smart transport." Pandian, M. Durai et al [12] presents the "Sleep Pattern Analysis and Improvement Using Artificial Intelligence and Music Therapy."

Bashar et al [13] elaborates the "Survey on Evolving Deep Learning Neural Network Architectures." Pandian, A. Pasumpon et al [14] puts forth the "Artificial Intelligence Application in Smart Warehousing Environment for Automated Logistics." J. Vijitha Ananthi et al [15] has designed an "Automation Using Iot in Greenhouse Environment."

\section{Proposed Work}

The expert system such as the genetic algorithm, artificial neural networks and the fuzzy systems are usual AI methods used in formulating a smart street lighting system, the ANN is preferred in the proposed system due to the malleable reconfiguration, of the frame, and the capability to acquaint with variety of inputs and further for its capability to train the developed neural networks and modify their parameters. The block diagram of the basic street light concept is shown in figure. 2 below.

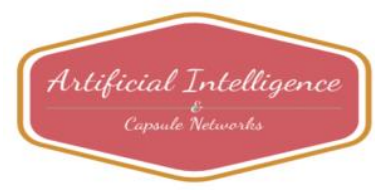


Journal of Artificial Intelligence and Capsule Networks (2020)

Vol.02/ No. 01

Pages: $42-52$

http://irojournals.com/aicn/

DOI: https://doi.org/10.36548/jaicn.2020.1.005

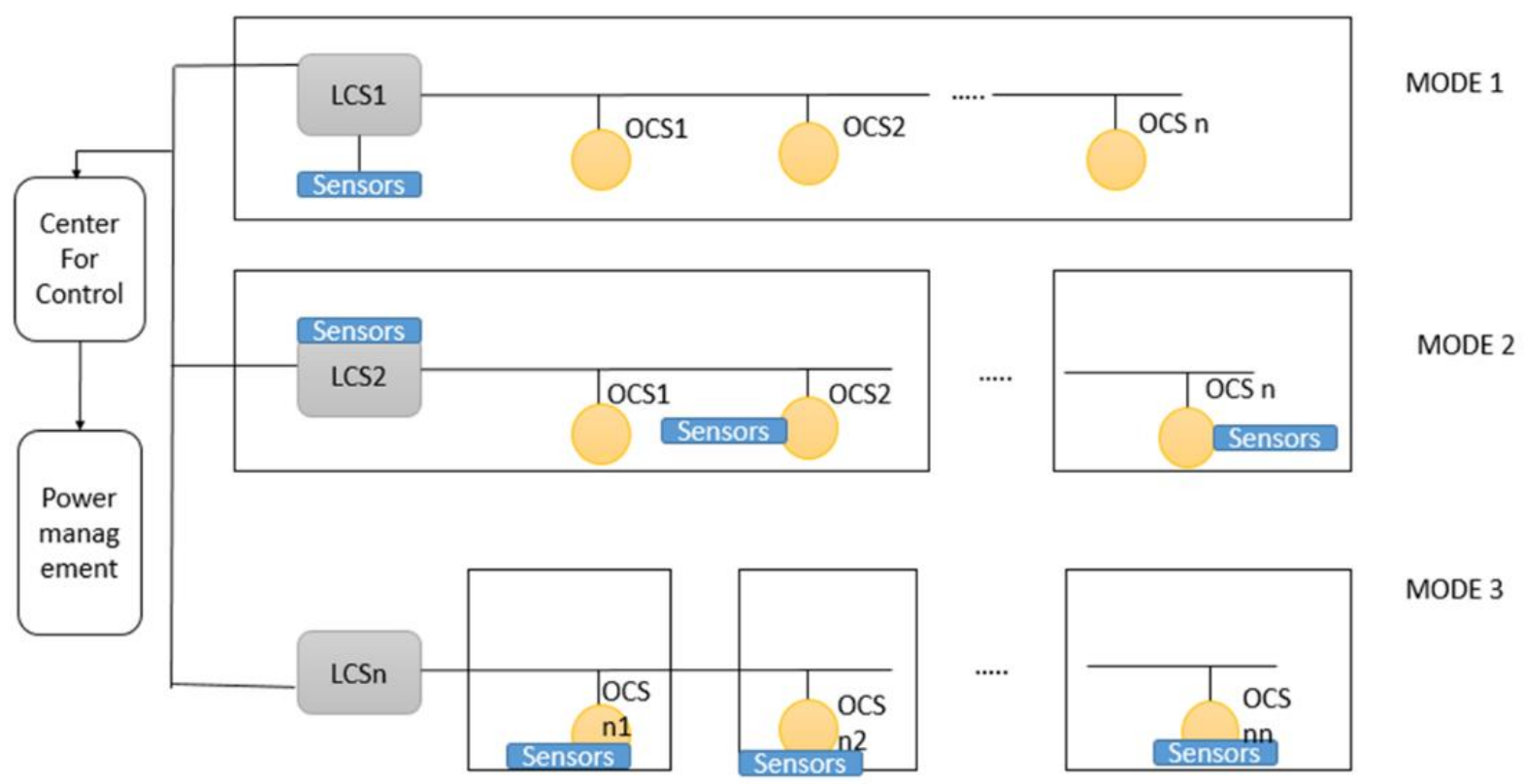

Figure.2 Basic Street Light Concept with ANN Control

The input data for training are usually formed based on the rain, temperature, cloudy, haze, atmospheric pressure and other details that are associated with the weather conditions. The system designed is capable of delivering two functions (i) prognostic functions- that is helpful in regulating the intensity of the light for the following twenty four hours. It is, by definition, a supporting device in which the values deciding the strength of lighting are compared to the values of the classical control system. The operator decides the device takes precedence-either traditional or intelligent, while the results obtained in this case are transmitted to the luminaire control units. (ii) The second feature is the continuous adjustment of the light intensity level for different electric light unit, depending on the current weather conditions. In this case, the set of sensors from which the values can be read at the NN input must be mounted in the specified electric light unit, lighting strings or control cabinets. Such values are used to correct the plan drawn up previously.

The intensity of the light is controlled by the proper communication that is extended with the control center. The center to control takes care of the various functions such as control-signal conveyance, to the strings of lighting, reception of particulars about the miscalculation done by multitudes of devices and goes through the prevailing weather forecast condition and further the prevailing weather conditions are sent to the Center for control. The Expert system devised takes in the information of the sensors and transmits the level of the

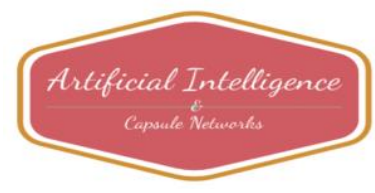


Journal of Artificial Intelligence and Capsule Networks (2020)

Vol.02/ No. 01

Pages: $42-52$

http://irojournals.com/aicn/

DOI: https://doi.org/10.36548/jaicn.2020.1.005

light intensity for every luminaire to the Center for control. Each strings of lighting are aided by the ANN and every strings holds at least 10 to 15 electric light unit s, this taken care by a linear control system-LCS in the higher level, Objective Control System-OCS in the lower. The LCS and OCS takes care of the proper arrangement of the lighting unit are holds responsibility in obtaining the values form the sensors that are located in the electric light unit.

The Three modes of operation of the is listed below in the table.1 provides below

\begin{tabular}{|l|l|}
\hline \multicolumn{1}{|c|}{ Modes of Operations } & \multicolumn{1}{|c|}{ Working } \\
\hline $\begin{array}{l}\text { Mode 1 } \\
\text { sensors are placed in the LCS }\end{array}$ & $\begin{array}{l}\text { This solution allows to control the overall } \\
\text { strings of lighting Neural Network has } \\
\text { only one output pointing out the level of } \\
\text { light for all the electric light units. }\end{array}$ \\
\hline $\begin{array}{l}\text { Mode } 2 \\
\text { electric light unit or a group of electric } \\
\text { light units in a given strings of lighting }\end{array}$ & $\begin{array}{l}\text { some conditions should be controlled in } \\
\text { other way than the remaining ones (like in } \\
\text { place of intersections, residential } \\
\text { streets etc.). LCS and OCS has own } \\
\text { Sensors, improved } \\
\text { individualization of the control process in } \\
\text { a given area, The electric units to be linked } \\
\text { are decided and regulated as prescribed. } \\
\text { Neural network has many outputs equal } \\
\text { the sections in the strings of lighting } \\
\text { learning process is prolonged as the } \\
\text { number of inputs and outputs are more } \\
\text { making the system complicated }\end{array}$ \\
\hline $\begin{array}{l}\text { Mode 3 } \\
\text { OCS has own set sensors }\end{array}$ & $\begin{array}{l}\text { This solution is least economic } \\
\text { due to the large number of sensors, but the } \\
\text { advantage is the } \\
\text { ability to control each electric light unit } \\
\text { separately. strings of lighting demands a } \\
\text { separate neural network }\end{array}$ \\
\hline
\end{tabular}

Table.1 Operation Modes

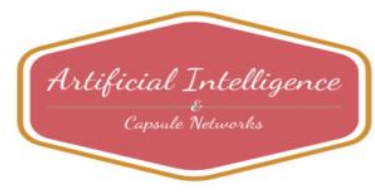


Journal of Artificial Intelligence and Capsule Networks (2020)

Vol.02/ No. 01

Pages: 42-52

http://irojournals.com/aicn/

DOI: https://doi.org/10.36548/jaicn.2020.1.005

To fashion a general solution, some generalizations has to be done for all three modes. The generalized model would hold neural networks with multiple outputs according to the Electric light units available in the strings of lighting and inputs range similar to the number of data sensed from the sensors in the OCS. If the One OCS fails to provide the input, the input is received from the next OCS of the same section. The data fed as input only from the strings of lighting. The data count is duplicated according to the number of electric light unit and as well as the OCS.

\section{Results Evaluation}

The experiment starts with the mode one operation, such that the neural network for tells the intensity level of all the lights, in a same fashion. The NN was trained with the meteorological information's with the single weather station situated in the city so its initial phase performance, with the power management was not much convenient and this led to the next two modes of operation. These modes utilized the feed forward neural networks with the back propagation and the momentum factor. The training process was equal to the number of hidden layer in the network. All the examinations were done on 20 cycles of learning and with each cycle having an iteration of 200 to 40,000. Initial weight values for specific neurons were randomly selected and were close to zero. For a given number of neurons in this layer, it is important to perform at least a dozen simulations to avoid stopping the error function at a local minimum. The dataset for training is encompassed with 9000 patterns acquired from different weather stations located in three various cities. The data selected was segregated into three datasets for testing, training and validating, the training was stopped when a mean square error was reached as the training process similar to .1 to .9 is opted. The results below in figure .3 shows the predicted error obtained in the process of training with 200 epochs

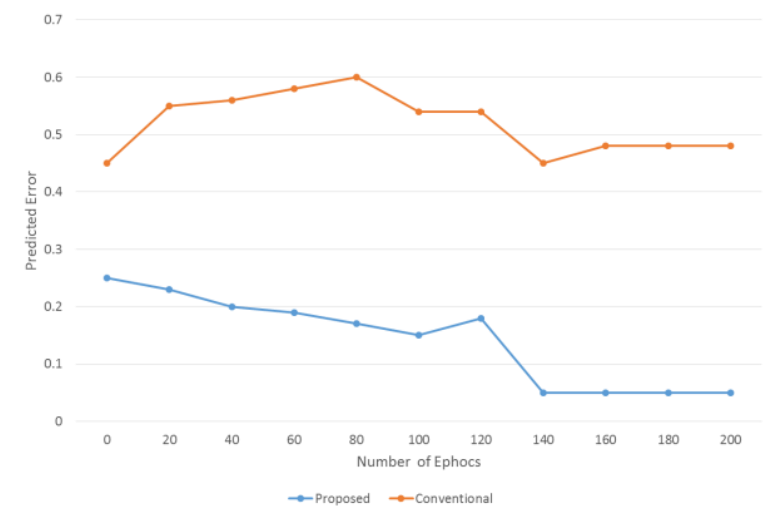

Figure.3 Prediction error

49

ISSN: 2582-2012 (online)

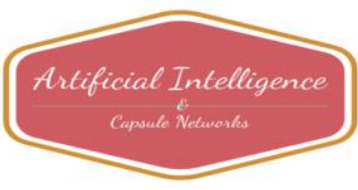


Journal of Artificial Intelligence and Capsule Networks (2020)

Vol.02/ No. 01

Pages: $42-52$

http://irojournals.com/aicn/

DOI: https://doi.org/10.36548/jaicn.2020.1.005

The results depicted in figure.4 shows the ANN are capable of efficiently expediting the procedure of the power management in the street light, diminishing the energy expended, the power reduction on the proposed and the conventional systems explained above are reported in figure. 4

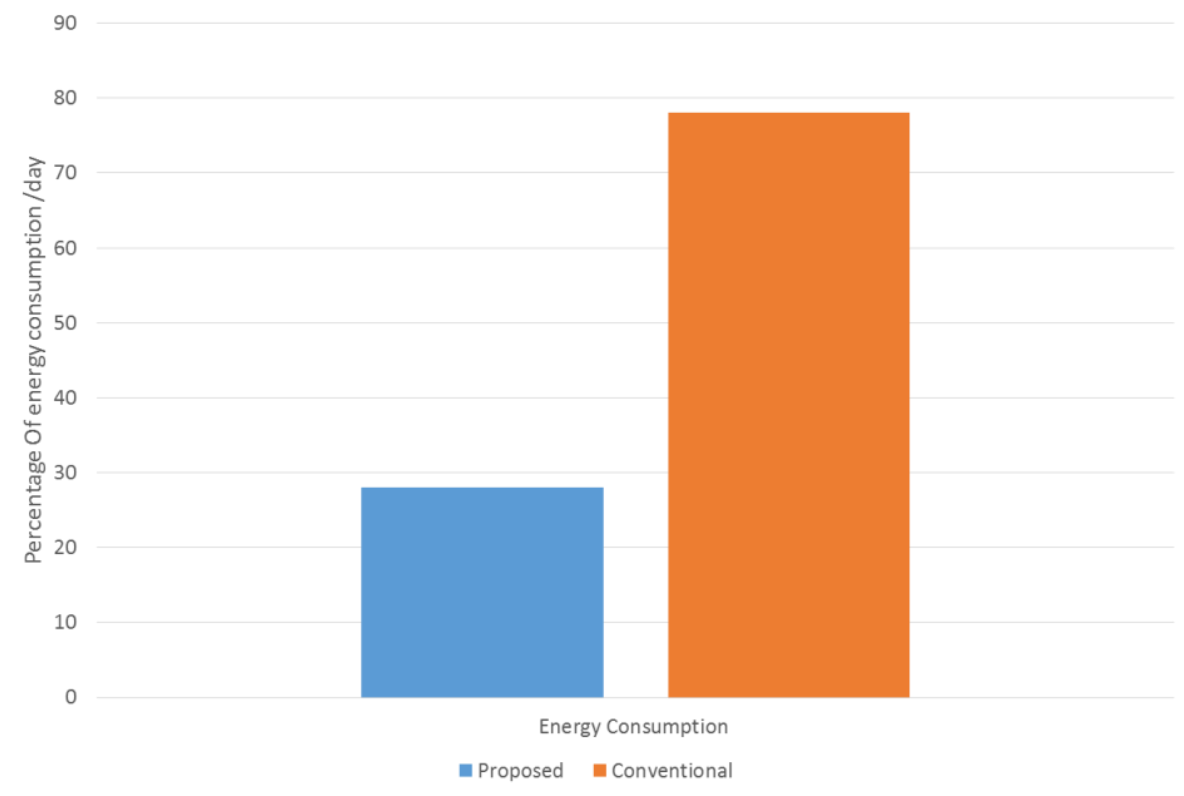

Figure.4 Energy Consumption

\section{Conclusion}

The Smart lighting system for the streets founded on the ANN is proposed in the paper, the program can be combined with the complete observing and regulator method for street lighting this is an emphasis of the bigger assignment. The system's function is to boost power competence by lowering the intensity of light, depending on the authentic environment. These remedies helps to reduce the cost of energy. The research is one of the phases of bigger assignment and thus the studies offered ought to be treated equally preliminary. As for the actual operation of the device, the findings obtained so far will have to be checked. They are prepared for future extension in the metro cities. Using other variables into the training vector would also help refine the proposed methodology and this is to be proceeded as the future work of the paper.

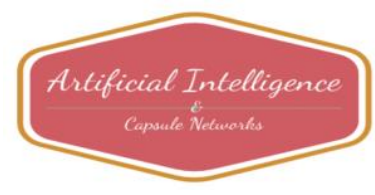


Journal of Artificial Intelligence and Capsule Networks (2020)

Vol.02/ No. 01

Pages: $42-52$

http://irojournals.com/aicn/

DOI: https://doi.org/10.36548/jaicn.2020.1.005

\section{References}

[1] Canca, D., J. Larrañeta, S. Lozano, and L. Onieva. "Traffic intensity forecast in urban networks using a multilayer perceptron." In Joint International Meeting EURO XV-INFORMS XXXIV. Barcelona, Spain. 1997.

[2] Ishak, Sherif, Prashanth Kotha, and Ciprian Alecsandru. "Optimization of dynamic neural network performance for short-term traffic prediction." Transportation Research Record 1836, no. 1 (2003): 45-56.

[3] Volosencu, Constantin, Daniel Ioan Curiac, Ovidiu Banias, Cristian Ferent, Dan Pescaru, and Alexa Doboli. "Hierarchical approach for intelligent lighting control in future urban environments." In 2008 IEEE International Conference on Automation, Quality and Testing, Robotics, vol. 1, pp. 158-163. IEEE, 2008.

[4] Rea, Mark S., John D. Bullough, and Yukio Akashi. "Several views of metal halide and highpressure sodium lighting for outdoor applications." Lighting Research \& Technology 41, no. 4 (2009): 297-320.

[5] .Pamuła, Teresa. "Road traffic parameters prediction in urban traffic management systems using neural networks." Transport Problems 6 (2011): 123-128.

[6] Saravanan, K., N. Muthu Prabhu, and B. Raja Rajeswari. "Fuzzy controller design of lighting control system by using VI package." International Journal of Artificial Intelligence 1, no. 2 (2012): 73-78.

[7] Ramadhani, Farah, Kamalrulnizam Abu Bakar, and Muhammad Gary Shafer. "Optimization of standalone street light system with consideration of lighting control." In 2013 The International Conference on Technological Advances in Electrical, Electronics and Computer Engineering (TAEECE), pp. 583-588. IEEE, 2013.

[8] Kumar, R. Praveen, and S. Smys. "A novel report on architecture, protocols and applications in Internet of Things (IoT)." In 2018 2nd International Conference on Inventive Systems and control (ICISC), pp. 1156-1161. IEEE, 2018.

[9] Raj, Jennifer S. "A Comprehensive Survey on the Computational Intelligence Techniques and Its Applications." Journal of ISMAC 1, no. 03 (2019): 147-159.

[10] Bestak, Robert, and S. Smys. "Big Data Analytics for Smart Cloud-Fog Based Applications." Journal of trends in Computer Science and Smart technology (TCSST) 1, no. 02 (2019): 74-83.

[11] Koresh, M. H., and J. Deva. "Computer vision based traffic sign sensing for smart transport." J. Innovative Image Process.(JIIP) 1, no. 01 (2019): 11-19.

[12] Pandian, M. Durai. "Sleep Pattern Analysis and Improvement Using Artificial Intelligence and Music Therapy." Journal of Artificial Intelligence 1, no. 02 (2019): 54-62.

[13] Bashar, Abul. "Survey on Evolving Deep Learning Neural Network Architectures." Journal of Artificial Intelligence 1, no. 02 (2019): 73-82.

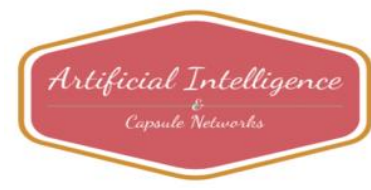


Journal of Artificial Intelligence and Capsule Networks (2020)

Vol.02/ No. 01

Pages: $42-52$

http://irojournals.com/aicn/

DOI: https://doi.org/10.36548/jaicn.2020.1.005

[14] Pandian, A. Pasumpon. "Artificial Intelligence Application in Smart Warehousing Environment for Automated Logistics." Journal of Artificial Intelligence 1, no. 02 (2019): 6372.

[15] Raj, Jennifer S., and J. Vijitha Ananthi. "Automation Using Iot in Greenhouse Environment." Journal of Information Technology 1, no. 01 (2019): 38-47. 